\title{
Development of CdS/CdTe Diode for X-Ray Sensor
}

\author{
H. SHAMS ${ }^{1}$, H. ABOU GABAL ${ }^{1}$, M. SOLIMAN ${ }^{2}$, S. EBRAHIM ${ }^{2}$, S. AGAMY ${ }^{1}$ \\ ${ }^{1}$ Nuclear and Radiation Engineering Department, Faculty of Engineering, Alexandria University, \\ Alexandria, EGYPT. \\ ${ }^{2}$ Materials Science Department, Institute of Graduate Studies and Research, Alexandria University, \\ Alexandria, EGYPT.
}

\begin{abstract}
Many methods are used to detect x-ray are incapable of accounting for the high x-ray flux generated by modern x-ray Instruments. The major technology for measurement of x-ray dose rate in real time is the ionizing chambers detectors, but it has some disadvantages like complexity. Also it has large size due to the importance of gas volume and pressure, high voltages, signal cables, and other specialized parts needed for its operation. Advances in the technology of CdTe semiconductor in solar cells industries allow the development of an inexpensive and compact solid-state X-ray sensor. As X-ray photons pass through the diode, the photoelectric effect produces a photocurrent. The X-ray flux can be determined from this current. Four stacked diodes were connected in series to measure the device performance. It was observed that amplitude of the pulse formed due to exposed FTO/CdS/CdTe/Mo detector to X-ray of 33 $\mathrm{keV}$ and $1 \mathrm{~mA}$ intensity is $1.03 \mathrm{~V}$.
\end{abstract}

Keywords: CdTe; Diode; X-ray; Sensor.

Received: June 12, 2020.Revised: November 4, 2020. Accepted: November 15, 2020. Published: December 11, 2020

\section{Introduction}

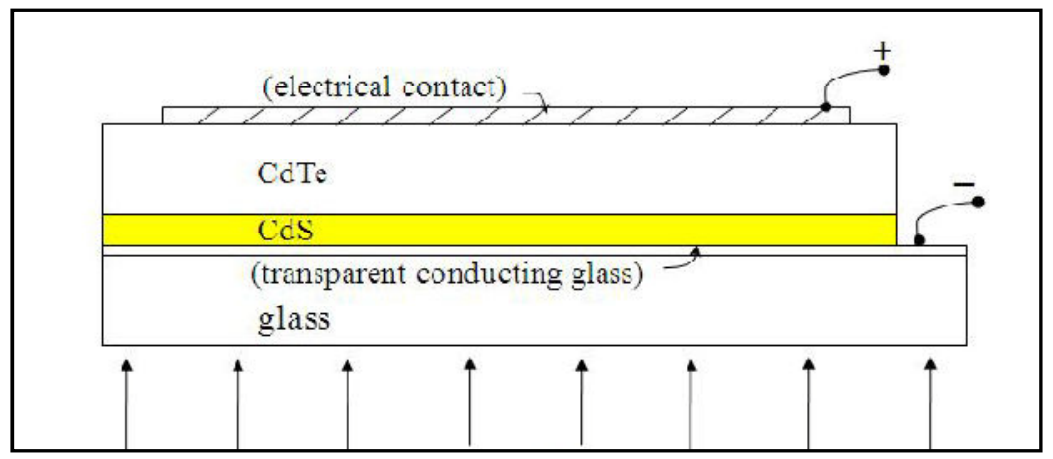

Figure 1: Layers of the diode. 
The different layers that compose a thin layer detector are shown in figure (1) and are described briefly below. Transparent conducting films are thin films which are transparent to light and conduct electricity. They are one of the main constituents of photovoltaic solar cells [1]. They work as a window that the light traverses to interact with the active material (absorber) where carrier generation occurs as shown in Figure (1). They also work as an ohmic contact for the transport of charge carriers out of the photovoltaic. The transparent materials have band gaps with energies corresponding to wavelengths shorter than the visible range of $380 \mathrm{~nm}$ to $750 \mathrm{~nm}$. To fabricate a practical radiation sensor, a means is needed to collect the electrical charge carriers from the semiconductor material. An ohmic contact will work as an electrode through which charges of both signs can go easily. When two ohmic contacts are provided at the opposite faces of a semiconductor slab, and connected to a measuring readout circuit, equilibrium in the concentrations of the charge carriers in the semiconductor is sustained. If an electron is collected at one electrode, another electron will be injected at the opposite electrode to keep the equilibrium concentration in the semiconductor, the same mechanism occurs for the holes [2]. The collection of charges from the semiconductor electrodes needs application of a high voltage. The high voltage will cause the production of a leakage current, even with the highest resistivity material available. As an alternative, blocking electrodes can be used to reduce the leakage current which passes through the semiconductor. When they are applied, the charge carriers initially collected from one electrode will not be replaced at the opposite electrode, so the charge concentration within the semiconductor will drop after application of an electric field. The leakage current will be reduced to a low level which allows the detection of any additional charges created by the radiation. The best type of blocking electrodes is the $p-n$ semiconductor. Any movement of electrons from the p-type semiconductor will face high resistance because the holes are the majority and free electrons are relatively rare. On the contrary, in the n-type semiconductor electrons are the majority carriers and the holes are rare, so the holes will face high resistivity to be injected from the $\mathrm{n}$ side [3].

Radiation Absorption layer It is a semiconductor material with suitable thickness sufficient to stop most of the incident radiation where electron-hole pairs are produced in the sensitive volume of the absorber layer. Under the effect of an electric field the produced charges will be collected through the electrodes [4].

Figure (1) shows the structure of the sensor fabricated in this work. It consists of:

- glass substrate coated with conducting material as fluorinated tin oxide,

- Thin film of n-type semiconductor as $\mathrm{CdS}$ that serves as a blocking contact,

- Thin film of p-type semiconductor as CdTe that serves as the absorber layer for the incident radiation and where the electron hole pair is produced and finally

- ohmic contact, for example molybdenum layer.

The aim of this work is to prepare an Xray sensor in which $\mathrm{Mo} / \mathrm{CdTe} / \mathrm{CdS} / \mathrm{FTO}$ devices are stacked together and operated as a single sensor. The work focused on the deposition and characterization of polycrystalline thick films of $\mathrm{CdS}$ as n-type and CdTe as p-type to obtain optimum parameters for the preparation of uniform and homogenous polycrystalline films with reasonable thickness for X-ray sensing, in addition to testing the fabricated detector. 


\section{Materials and Methods}

\subsection{Materials}

Cadmium chloride pentahydrate was obtained from Sigma Aldrich. Cadmium sulfate hydrate $(98 \%, \mathrm{MW}=769.51 \mathrm{~g} / \mathrm{mole})$ was obtained from Merck, Tellurium dioxide $(99 \%, \mathrm{MW}=159.6 \mathrm{~g} / \mathrm{mole})$ was obtained from Sigma Aldrich, Fluorine doped tin oxide (FTO) coated glass slide with sheet resistance of 7 $\mathrm{ohm} / \mathrm{sq}$ and Transmittance of $80-82 \%$ (visible), was bought from Sigma Aldrich.

\subsection{Preparation and thermal chemical treatment of CdTe thin films}

CdTe films had been deposited by potentiostat electro deposition and carried out using a 2-electrode cell according to the comparative study of O. K. Echendu et al [5]. Potentiostat controls the voltage between reference and working electrodes while the device is passing the current through a counter electrode. So the potentiostat is controlling the voltage and measuring the change in the passing current.

The electrodeposition system consists of glass/FTO working electrode as cathode and a graphite electrode as anode immersed in an electrolyte. The aqueous electrolyte solution was prepared by Cadmium sulfate $(3.8 \times 10-2 \mathrm{M})$ solution, $(4.2 \times 10-2 \mathrm{M})$ of $\mathrm{CdCl} 2,(10-3 \mathrm{M}$ and $2 \times 10-3 \mathrm{M})$ of $\mathrm{TeO} 2$. The $\mathrm{pH}$ was then adjusted to $2.00 \pm 0.02$ using high purity $\mathrm{H}_{2} \mathrm{SO}_{4}$. The produced films have been chemically heat treated at $350 \mathrm{oC}$ for 15 min with $1 \mathrm{~N} \mathrm{CdCl}_{2}$.

\subsection{Fabrication of CdS/CdTe X-ray detector}

CdTe was electrodeposited onto prepared CdS/FTO substrates. Molybdenum thin films were produced on $\mathrm{CdTe} / \mathrm{CdS}$ FTO glass substrates using RF sputtering system. The substrates were rinsed in running distilled water and dried using oven. They were subsequently introduced into the vacuum chamber and placed at a distance of $10 \mathrm{~cm}$ from the Mo target. The vacuum chamber was initially evacuated to a base pressure of $6 \times 10^{-4}$ Torr. Then pure Argon $(99.999 \%)$ flow of 0.094 standard liter per minute (SLPM) was introduced into the chamber to keep the pressure during the film deposition at a value around $1.45 \mathrm{mTorr}$. The power supply was then turned on. The power was adjusted at $60 \mathrm{~W}$. A series of sixteen Mo films back contact was prepared, according to the study of molybdenum thin film deposition by N.Dhara et al [6].

The schematic diagram of the fabricated detector with different layers is shown in Figure (2).

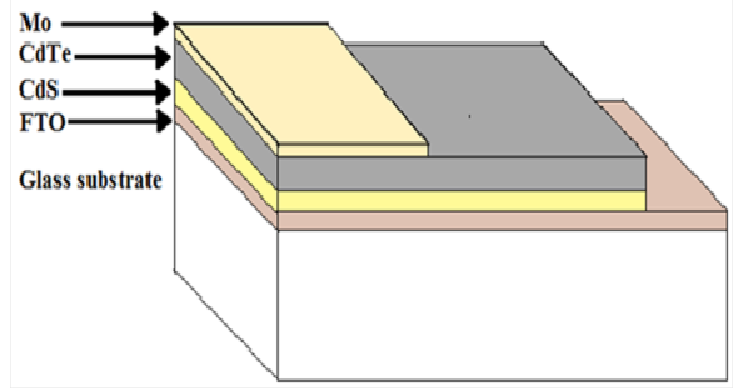

Figure : 2 The structure of the fabricated X-ray sensor. 


\subsection{Measurement of X-ray Sensor Performance}

X-ray generator Geratogram Roentgen instrument figure (3) had been made in Germany 1987 , is used to produce X-ray with energy adjustable range of 21 to $42 \mathrm{keV}$ (wave length $\lambda$ range 29.5 to 59.0 picometer, pm). Also its emission intensity is adjustable in the range from 0.05 to $1 \mathrm{~mA}$, the local dose rate at a distance of $0.1 \mathrm{~m}$ from touchable surface of the device is $\leq$ $5 \mu \mathrm{Sv}[7]$.

To enhance the exposed area of the sensor, we stacked four sensors in series. The positive electrode of first sample (Mo) is connected directly to the negative electrode of the second sample (FTO) and so on. The sensor performance is investigated by exposing the stacked sensor directly perpendicular to the Xray source. The output signal of the stacked sensor was connected to computerized oscilloscope DS03064 Kit V.

\section{Results and Discussion}

The signal pulse generated from a single event depends on the input characteristics of the circuit, e.g. a preamplifier, to which the detector is connected. Figure (4) shows the equivalent input circuit where $\mathrm{R}$ is the input resistance of the circuit, and $\mathrm{C}$ is the equivalent capacitance of the detector and the measuring circuit.

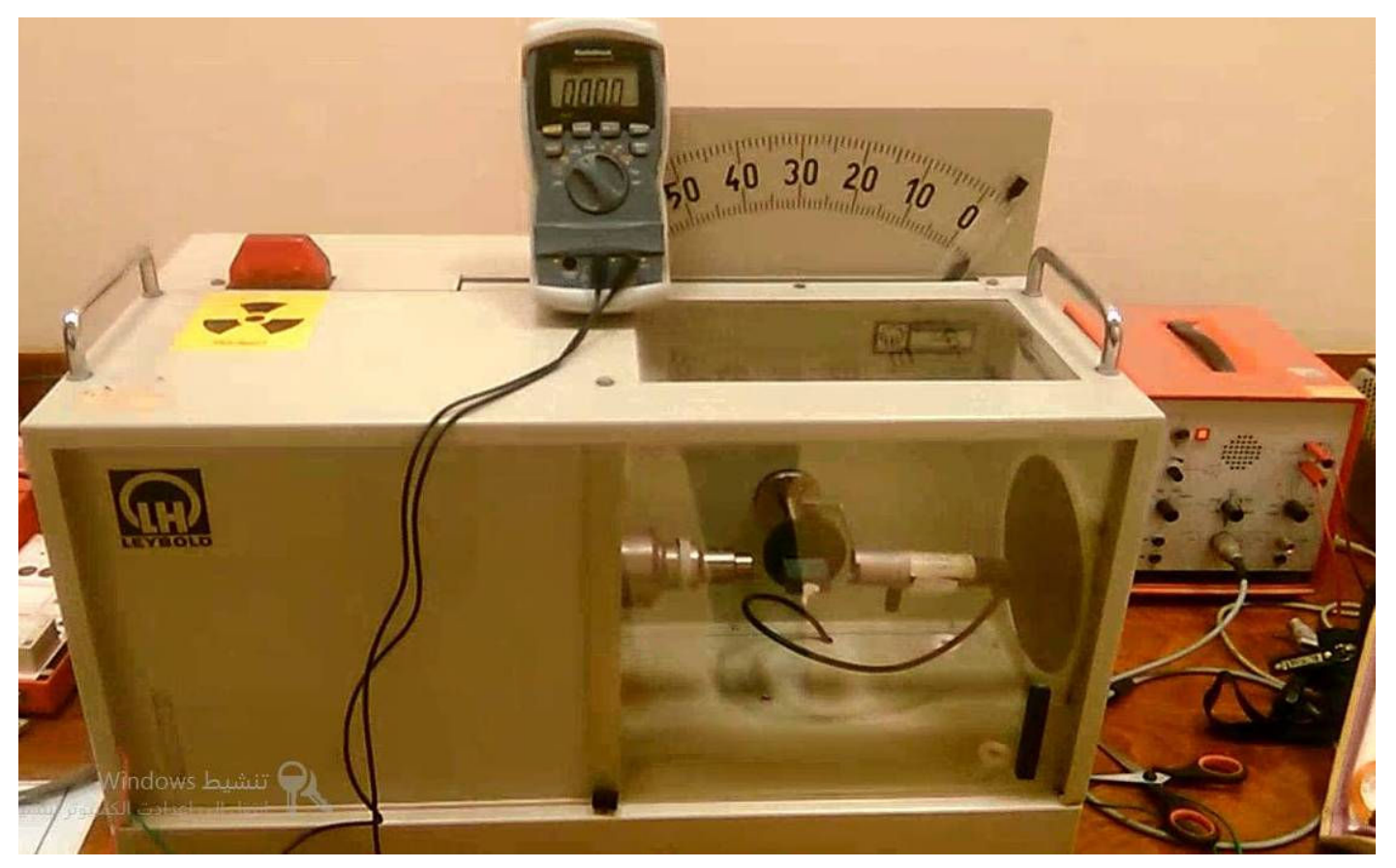

Figure : 3 X-ray generator Geratogram Roentgen instrument. 

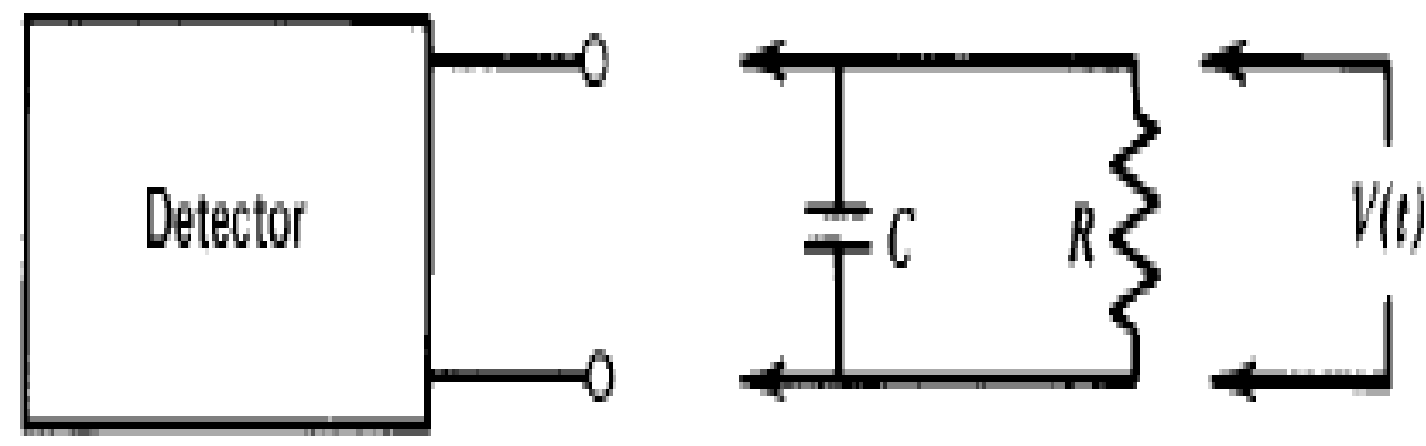

Figure 4: Pulse mode circuit [8].

If we assume that $\mathrm{V}(\mathrm{t})$ is the voltage as function of time across the load resistance. This voltage is the basic signal voltage on which the pulse mode operation is based. We have two cases of operation which depend on the time constant of the measuring circuit. This time constant is simply calculated by the following equation:

$$
z=R C
$$

First case: very low value of time constant $\mathrm{Z}$.

Here the time constant of the circuit is assumed to be very small in comparison with the time of charge collection $t_{c},\left(z \ll t_{c}\right)$. In this case, the output pulse voltage $V(t)$ will have the signal voltage $V(t)$ nearly identical to the time dependence of the current produced within the detector as illustrated in Figure $(5 a, b)$. It is used in some cases where the radiation rate is too high, and the counting of events is more important than obtaining the energy information. constant $\mathrm{z}$.

Second case: very high value of time

This case is more general, where the time constant of the circuit is assumed to be very large in comparison with the time of charge collection $t_{c},\left(z \gg t_{c}\right)$. According to this assumption the time between pulses is large enough, so the discharge of capacitance will be done through the resistance and the voltage returns to zero value. The output signal voltage $\mathrm{V}(\mathrm{t})$ is shown in figure $(5 \mathrm{c})$. The main factor in determining the time required for the pulse to reach its maximum is the time of charge collection and does not depend on the load circuit. But the time needed for the signal voltage to return to zero depends only on the load circuit. So, the signal head edge depends on the detector, but the signal tail edge depends on the circuit. This basic case is very common in a lot of radiation detectors which operate with pulse mode. The maximum voltage $V_{\max }$ which is the signal amplitude can be calculated by dividing $\mathrm{Q}$ (total collected charge) by $\mathrm{C}$ (capacitance). The capacitance is constant, so the signal amplitude will depend only on the collected charge.

$$
V_{\max }=\frac{Q}{C}
$$


The output of the pulse mode detector is given as separate distinct pulses. The individual pulse is related to specific interaction between the incident radiation and the detector. The count of the pulse rate is a measure of the rate of the radiation interactions inside the detector. For calculation of the radiation energy we measure the pulse amplitude because it is directly proportional to the collected charge. We can measure the energy distribution of the radiation by measuring the variation in the amplitude of the output signal. If the capacitance stays constant the proportionality between the collected charge and amplitude will hold. In the real case the capacitance of semiconductor usually changes with the working conditions. This will cause pulses with different amplitude for radiation with the same collected charge Q. to solve this problem and keep the proportionality, we use specific circuit called charge sensitive circuit. It is a circuit that contains a preamplifier, which can eliminate the effect of capacitance variation and keep the proportionality between the collected charge Q and the pulse amplitude [8].

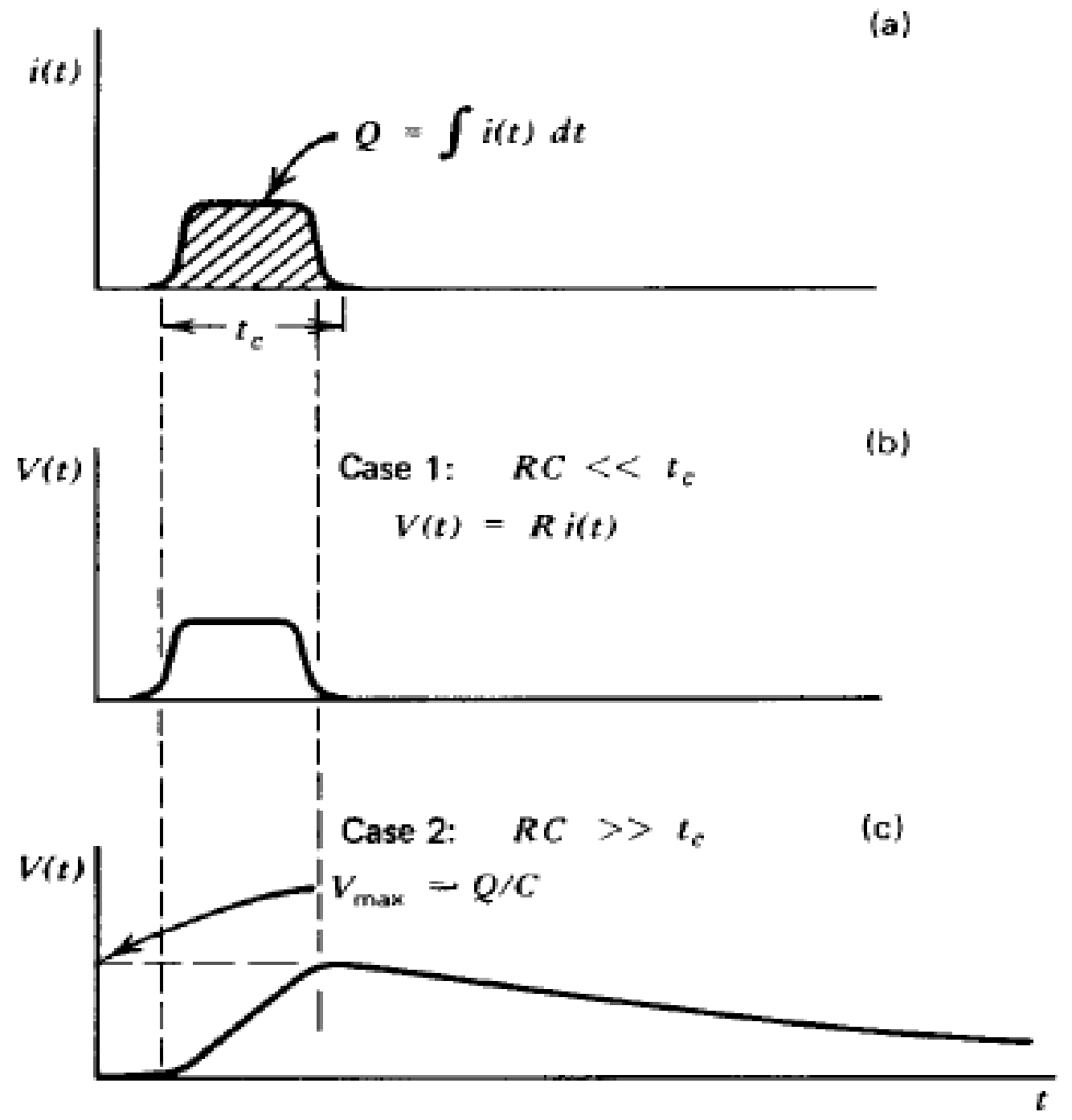

Figure 5: Voltage as function of time in pulse mode (a) $\mathrm{z}<<$ tc (b) $\mathrm{z}>>$ tc (c) $\mathrm{V} \max [8]$. 
Figures $(6 \mathrm{a}, \mathrm{b}, \mathrm{c})$ show output signal from $\mathrm{FTO} / \mathrm{CdS} / \mathrm{CdTe} / \mathrm{Mo}$ sensor exposed to X-ray of 21,27 and $30 \mathrm{keV}$ at $0.05 \mathrm{~mA}$ intensity. It is observed that the maximum amplitudes of the pulse formed due to the exposure of $\mathrm{FTO} / \mathrm{CdS} / \mathrm{CdTe} / \mathrm{Mo}$ sensor to X-ray are 0.41 , 0.65 and $0.72 \mathrm{~V}$ for the accelerated voltage 21,27 and $30 \mathrm{kV}$ respectively. Figures $(7 \mathrm{a}, \mathrm{b}, \mathrm{c})$ show output signal from $\mathrm{FTO} / \mathrm{CdS} / \mathrm{CdTe} / \mathrm{Mo}$ sensor exposed to X-ray of 21,27 and $33 \mathrm{keV}$ at $1 \mathrm{~mA}$ intensity. It is observed that the maximum amplitudes of the pulse formed due to the exposure of $\mathrm{FTO} / \mathrm{CdS} / \mathrm{CdTe} / \mathrm{Mo}$ sensor to X-ray are $0.64,0.86$ and $1.03 \mathrm{~V}$ respectively. The produced peaks with X-ray exposure can be explained by using the equivalent electrical circuit of the detector. The circuit resistance of the $\mathrm{FTO} / \mathrm{CdS} / \mathrm{CdTe} / \mathrm{Mo}$ sensor and the measuring circuit is assumed to be $\mathrm{R}$, while the total capacitance assumed to be $\mathrm{C}$. The voltage $\mathrm{V}(\mathrm{t})$ across the load resistance as a function of time is the main output signal voltage. The time constant of the circuit could be simply calculated by RC. Assume that the time separate pulses is large enough, so the discharge of the charged capacitance will be performed via the resistance as mention by Knoll [8]. After discharge the voltage return to zero across the load resistance. In this case the main factor which determine the time required for the signal pulse to reach its maximum value is the charge collection time in the $\mathrm{FTO} / \mathrm{CdS} / \mathrm{CdTe} / \mathrm{Mo}$ sensor. Although the time required to retain the signal voltage to zero is affected by the time constant of the load circuit. We can calculate the pulse amplitude by dividing the total charge created in the detector during one interaction by the capacitance of load circuit. Assuming that the capacitance of the load circuit is constant as indicated by Knoll [8], so the amplitude of the signal pulse is only proportional to the total collected charge Q. The signal formation can be explained through three steps [27]. The first step, the formation of depletion region or junction prevents the passing of the free charges from the bulk. X-ray passes through the FTO/CdS/CdTe/Mo sensor. X-ray photons disappeared, and a photoelectron ejected from one of the inner electron shells of the absorber $\mathrm{CdS} / \mathrm{CdTe}$ sensor. The ejected electron will hold kinetic energy equals to the incident photon energy minus the binding energy of the electron in its original shell. The second step, The vacancy that is formed in the electron shell as a result of the photoelectron ejection is refilled very fast in an electron rearrangement inside the atom, this rearrangement will cause a characteristic X-ray is produced. The third step, $\mathrm{X}$-rays reabsorbed again through photoelectric interactions with less tightly bound electron shells of the $\mathrm{CdS} / \mathrm{CdTe}$ junction. the produced electrons make collisions in its path with the absorber electrons causing the appearance of a given amount of electric charge within the detector. These created charges flow in the opposite directions and collected at the electrodes causing electrical pulses. The obtained results is with good agreement with the result obtained by J. Abramovitch [9].

Figure (8) shows the output signal of $\mathrm{FTO} / \mathrm{CdS} / \mathrm{CdTe} / \mathrm{Mo}$ sensor versus X-ray energy at different intensities. The amplitude is linearly increased with energy of X-ray where we can predict three regions. Region one from 21 to 27 $\mathrm{KeV}$ where there is high sensitivity to increase in $\mathrm{X}$-ray energy due to increase in number of electron-hole pair produced. Region two from 27 to $36 \mathrm{KeV}$, which there is equilibrium between electron-hole pair production and recombination, so the rate of increase in amplitude is reduced. Region three from 36 to $42 \mathrm{KeV}$, where saturation occur. The increase in amplitude not significant. The electron-hole pairs produced from high energy X-ray are far from the junction of the detector and consequently the recombination process of electron-hole pairs is dominant. We can see that the linearity of the $\mathrm{FTO} / \mathrm{CdS} / \mathrm{CdTe} / \mathrm{Mo}$ sensor is better at lower intensity of X-ray than that at higher intensity. In this work we used the energy range from 21 to $42 \mathrm{KeV}$ because the maximum obtained 
thickness for $\mathrm{CdS} / \mathrm{CdTe}$ junction is of range 4 $\mu \mathrm{m}$. The stacked four $\mathrm{CdS} / \mathrm{CdTe}$ junction connected in series increases the area of the sensor. As the area increases, the number of interactions of X-ray with $\mathrm{CdS} / \mathrm{CdTe}$ junction is enhanced resulting in the increase of the collected charges and the signal amplitude.

\section{Conclusion}

CdTe was electrodeposited onto the prepared $\mathrm{CdS} / \mathrm{FTO}$ substrates. The cyclic voltammetry was used to provide the optimum applied voltage for CdTe deposition onto $\mathrm{CdS} / \mathrm{FTO}$ glass substrate, where $-1.3 \mathrm{~V}$ was found suitable for CdTe film deposition. By growing CdTe layer onto $\mathrm{CdS} / \mathrm{FTO}$ at constant voltage a homogeneous good distribution layer was established with maximum thickness $4 \mu \mathrm{m}$. Mo metal as a back contact was deposited on $\mathrm{CdTe} / \mathrm{CdS} / \mathrm{FTO}$ hetero junction samples by DC magnetron sputtering system. The performance of the $\mathrm{FTO} / \mathrm{CdS} / \mathrm{CdTe} / \mathrm{Mo} \mathrm{X}$-ray detector was measured by computerized oscilloscope. The output electric were measured at 21, 27 and 30 $\mathrm{keV}$ and $0.05 \mathrm{~mA}$ intensity and were found to be $0.41,065$ and $0.72 \mathrm{~V}$ respectively, while the peak amplitude at 21,27 and $33 \mathrm{keV}$ and $1 \mathrm{~mA}$ intensity were found to be $0.64,0.86$ and $1.03 \mathrm{~V}$ respectively, which indicating good performance, response and sensitivity to different X-ray energy and intensity. By utilizing peak amplitude obtained from the detector, we demonstrate a new method to measure X-ray. with the 4 detector stacked in series. Stacked FTO/CdS/CdTe/Mo X-ray detector have been developed, demonstrating room-temperature semiconductor radiation detectors. The used fabrication method intended to be simple, low cost and available to reduce the production cost of the detector. This new sensor technology is useful for many applications requiring direct, high sensitive imaging of $\mathrm{X}$-rays at roomtemperature.

References:

1- A Bhubaneswari Paridaa, S. Iniyanb, Ranko Goicc, "Review of Solar Photovoltaic Technologies", Renewable and Sustainable
Energy Reviews 15, pp. 1625-1636, doi:10.1016/j.rser.2010.11.032, 2011.

2- S. M, SZE, "Semiconductor Devices Physics and Technology", Second Edition, John Wiley \& Sons, INC., 605 Third Avenue, New York, NY, ISBN 0-471-33372-7, Printed in the United States of America, Section 7.1, pp 225-237, 2002.

3- Nilofar Asim, Kamaruzzaman Sopian, Shideh Ahmadi, Kasra Saeedfar, M. A. Alghoul, Omidreza Saadatian, Saleem H. Zaidi, "A Review on the Role of Materials Science in Solar Cells", Renewable and Sustainable Energy Reviews 16 (2012), pp. 5834-5847, doi.org/10.1016/j.rser.2012.06.004, 2012.

4- Gordon R. Gilmore, "Practical gamma-ray spectrometry", 2nd Edition, John Wiley \& Sons, Ltd. ISBN: 978-0-470-86196-7, Warrington, UK, Chapter 3, pp. 39-59, 2008.

5- O. K. Echendu, K. B. Okeoma, C. I. Oriaku, and I.M. Dharmadasa, "Electrochemical Deposition of CdTe Semiconductor Thin Films for Solar Cell Application Using TwoElectrode and Three-Electrode Configurations: A Comparative Study"', Article ID 3581725, 8 pages, doi.org/10.1155/2016/3581725, 2016.

6- N. Dhara, P. Chelvanathana, M. Zamanb, K. Sopiana, N. Amina,b,c, "An Investigation on Structural and Electrical Properties of RFSputtered Molybdenum Thin Film Deposited on different Substrates", Energy Procedia, 33 (2013), pp. 186 - 197, doi: 10.1016/j.egypro.2013.05.057, 2013.

7- X-Ray Generator Geratogram Roentgen generate instrument $42 \mathrm{kV}$ Manual.

8- G. E. Knoll. Radiation Detection and Measurement 3rd edn., John Wiley and Sons, New York, PP. 48:53, 103:111, 308:317, 2000.

9- J. Abramovitch, Engaged learning at SMUD digital repository, Development of a Silicon PIN Diode X-Ray Detector (2014),http://digitalrepository.smu.edu/upjou rnal_research/32, 2014.

Creative Commons Attribution License 4.0 (Attribution 4.0 International, CC BY 4.0)

This article is published under the terms of the Creative Commons Attribution License 4.0

https://creativecommons.org/licenses/by/4.0/deed.en_US 

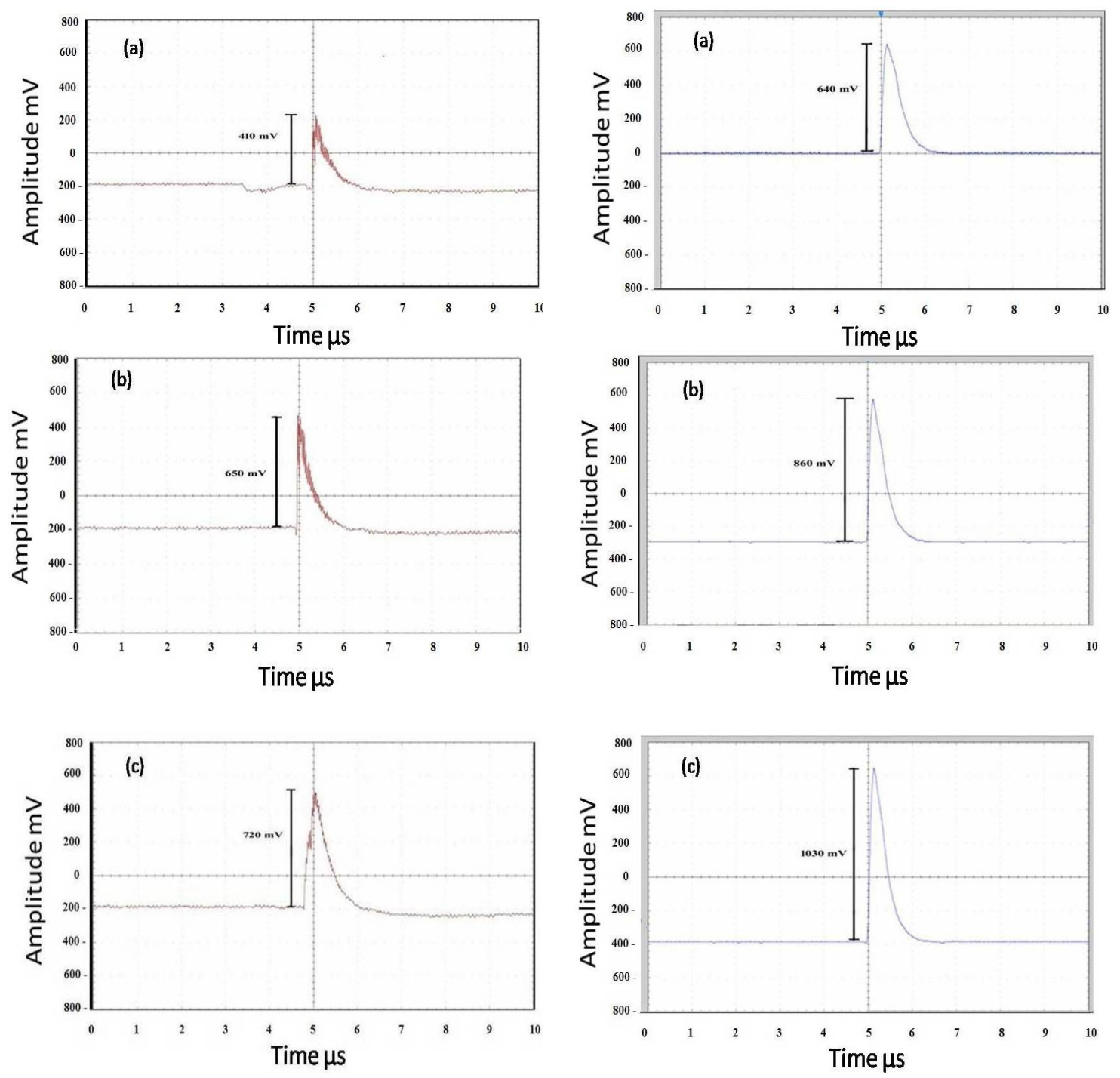

Figure 6

Figure 7

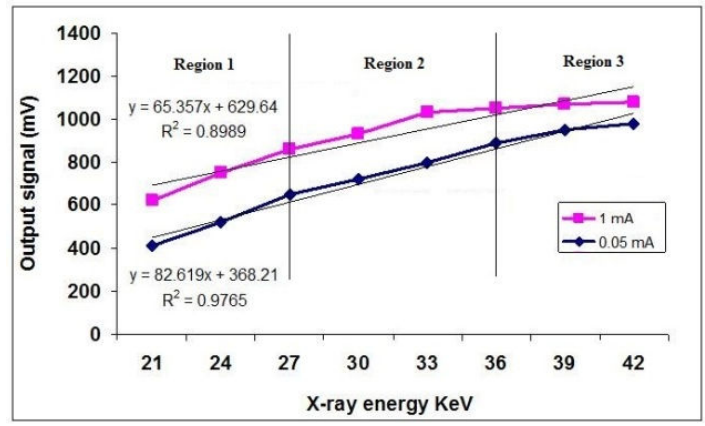

Figure 8 